\title{
Time-Resolved Speckle Analysis: A New Approach to Coherence and Dephasing of Optical Excitations in Solids
}

\author{
Langbein, Wolfgang Werner; Hvam, Jørn Märcher; Zimmermann, R.
}

Published in:

Physical Review Letters

Link to article, DOI:

10.1103/PhysRevLett.82.1040

Publication date:

1999

Document Version

Publisher's PDF, also known as Version of record

Link back to DTU Orbit

Citation (APA):

Langbein, W. W., Hvam, J. M., \& Zimmermann, R. (1999). Time-Resolved Speckle Analysis: A New Approach to Coherence and Dephasing of Optical Excitations in Solids. Physical Review Letters, 82(5), 1040-1043.

https://doi.org/10.1103/PhysRevLett.82.1040

\section{General rights}

Copyright and moral rights for the publications made accessible in the public portal are retained by the authors and/or other copyright owners and it is a condition of accessing publications that users recognise and abide by the legal requirements associated with these rights.

- Users may download and print one copy of any publication from the public portal for the purpose of private study or research.

- You may not further distribute the material or use it for any profit-making activity or commercial gain

- You may freely distribute the URL identifying the publication in the public portal 


\title{
Time-Resolved Speckle Analysis: A New Approach to Coherence and Dephasing of Optical Excitations in Solids
}

\author{
W. Langbein and J. M. Hvam \\ Mikroelektronik Centret, The Technical University of Denmark, Building 345 east, DK-2800 Lyngby, Denmark \\ R. Zimmermann \\ Institut für Physik der Humboldt-Universität zu Berlin, Hausvogteiplatz 5-7, D-10117 Berlin, Germany
}

(Received 11 September 1998)

\begin{abstract}
A new method to measure the time-dependent coherence of both homogeneously and inhomogeneously broadened optical excitations in solids is presented. The coherence degree of resonantly excited light emission is deduced from the intensity fluctuations over the emission directions (speckles). This method determines the decays of intensity and coherence separately, thus distinguishing lifetime from pure dephasing. The secondary emission of excitons in semiconductor quantum wells is investigated. Here the combination of static disorder and inelastic scattering leads to a partially coherent emission. The temperature dependence is well explained by phonon scattering. [S0031-9007(98)08270-2]

PACS numbers: 78.66.Fd, 42.25.Kb, 71.35.-y
\end{abstract}

Light emission from resonantly excited excitons in semiconductor nanostructures receives continued interest. In particular, the exciton dynamics after a short-pulse excitation and the temporal coherence of the emission are discussed in recent literature [1-6]. The conversion of the excitation light into a scattering (nonspecular) direction, which differs from the transmitted, reflected, or possibly diffracted directions, involves scattering. The degree of temporal and spatial coherence between the emitted and the exciting light fields thus contains information about the internal scattering processes, which include interface roughness, phonon emission, and exciton-exciton interaction. If static disorder dominates, the scattering is elastic and is called Rayleigh scattering, preserving the temporal coherence. The other extreme is (incoherent) luminescence, which has lost all phase memory. In time-resolved experiments, a distinction between the two cases is sometimes rather difficult. To avoid any preoccupation, the neutral notion of "secondary emission" has been introduced by others and will also be used here. Using the polarization and time-resolved secondary emission of excitons from a multiple quantum well (MQW) measured with a streak camera, Stolz et al. [1] deduced the coherent and incoherent intensities assuming polarization properties of these components. Femtosecond up-conversion experiments on MQWs by Wang et al. [2] and by Haacke et al. [3] measured the dynamics of the secondary emission intensity, but provide only indirect information about its nature considering the initial dynamics. Theory predicted a quadratic rise for the Rayleigh component, but a linear one for the luminescence [3]. More conclusive are interferometric correlation measurements. Two-pulse interferometric excitation with variable detection bandwidth used by Gurioli et al. [4] allows only for homogeneously broadened resonances to distinguish between the coherence in the excitation and the emission process. For inhomo- geneously broadened resonances, additional assumptions about the coupling between the resonances are necessary. Using a similar excitation, Wörner and Shah [6] time resolved the emission from an MQW with femtosecond upconversion and found three different components, from which two are related to the emission coherence but do not allow for a direct quantification. Interferometric measurements of the secondary emission by Birkedal and Shah [5] could demonstrate the partly coherent nature of the secondary emission. However, the spectral interferometry [7] used had limited spectral resolution, thus giving no reliable information for times longer than $\approx 10 \mathrm{ps}$. Additionally, the interferometric signal can be obtained only by detecting a single mode [5]. Otherwise, different phases of the emitted field are superimposed, suppressing the interferometric signal. The dynamics of different scattered modes differ substantially due to the statistical nature of Rayleigh scattering, demanding a statistical analysis of many modes. The corresponding intensity fluctuations over the emission direction (irregular pattern of bright and dark spots) are called speckles [8]. Using the intimate relation between coherence and speckles, we propose a novel method to measure the emission coherence: Instead of a two-pulse interferometry, which demands holographic stability, simply detect and analyze the speckle features in the emission. Time-resolved detection of speckles in the coherent nonresonant scattering has been recently reported [9] to analyze multiple elastic light scattering.

In the present work, we apply the speckle analysis to semiconductor optics, and time resolve the emission coherence after a short-pulse excitation. We present a quantification of the coherence by a statistical analysis of the speckle pattern. For the exciton system under study, the inhomogeneous broadening, the state lifetime, and the coherence decay by phonon scattering (pure dephasing) can be obtained. The method, however, is not restricted to 
this particular problem and can be applied to characterize the coherence of any secondary emission from solid-state materials. It gives simultaneous information about the intensity decay, characterized by the lifetime $T_{1}$, and the coherence decay, governed solely by the pure dephasing time $T_{2}^{\prime}$, and not influenced by $T_{1}$. It is based on a linear experimental method and is therefore quite sensitive. Other methods to determine the microscopic damping of inhomogeneously broadened resonances, such as spectral hole burning and photon echo [10] are nonlinear, limiting their applicability to high excitation densities.

The investigated samples are $\mathrm{GaAs} / \mathrm{Al}_{0.3} \mathrm{Ga}_{0.7}$ As single quantum wells (SQWs) with thicknesses of 35, 15, and $8 \mathrm{~nm}$, grown by molecular beam epitaxy. They are placed in a helium cryostat at a temperature varied between 5 and $40 \mathrm{~K}$. The fundamental hh1-e1 $1 s$ exciton resonance is excited by optical pulses from a mode-locked Ti:sapphire laser of 0.5 to 2 ps pulse length. The secondary emission in various directions is passed through a monochromator and detected by a synchroscan streak camera with a time resolution of 3 ps (see Fig. 1a). The angular resolution achieved by the second dimension of the streak camera was adjusted to resolve a single speckle, i.e., to the diffraction limit of the emission from the excited area on the sample. The spectral resolution of about $1 \mathrm{meV}$ rejects nonresonant emission, but does not deteriorate the temporal resolution. All presented data were taken with excitation in Brewster angle and detection normal to the sample, through an analyzer parallel to the linear excitation polarization.

The directional and temporal emission intensities, averaged over more than $10^{10}$ pulses, are shown in Fig. $1 \mathrm{~b}$ for a dominantly radiatively broadened excitonic transition (35 nm SQW), a transition with approximately equal homogeneous and inhomogeneous broadening ( $15 \mathrm{~nm} \mathrm{SQW})$, and a dominantly inhomogeneously broadened one $(8 \mathrm{~nm}$ $\mathrm{SQW}$ ). All three sets of data show strong variations along the scattering direction (speckle pattern). While this pattern is nearly constant in time for the $35 \mathrm{~nm}$ SQW, it ac- quires some temporal fluctuations for the $15 \mathrm{~nm} \mathrm{SQW}$, which become rather fast for the $8 \mathrm{~nm} \mathrm{SQW}$. Accordingly, the time-integrated intensity exhibits speckles only for the $35 \mathrm{~nm}$ SQW.

The time-dependent emission intensity $I(t)$ consists of a coherent and an incoherent part, $I=I_{\text {coh }}+I_{\text {inc }}$. We define the coherence $c$ of the emission as the fraction $c=\bar{I}_{\text {coh }} / \bar{I}$ where the average is taken over the scattering directions (at fixed time). While $I_{\text {inc }}$ has no significant variation over the direction, $I_{\text {coh }}$ fluctuates over the speckles. The latter can be viewed as a sum of field contributions with statistically independent phases since they are generated by uncorrelated scatterers (see below). According to the central limit theorem, a Gaussian distribution in the complex field plane evolves [11]. Consequently, the related (coherent) intensity will follow an exponential distribution. Adding the nonfluctuating incoherent part, we arrive at a displaced exponential distribution,

$$
p(I)=\theta(Z) \frac{1}{\bar{I}_{\text {coh }}} e^{-Z} \quad \text { with } Z=\left(I-\bar{I}_{\text {inc }}\right) / \bar{I}_{\text {coh }} .
$$

Experimental intensity histograms from the $8 \mathrm{~nm}$ SQW are analyzed in Fig. 2a. Displacement and asymmetry are obvious, as expected from Eq. (1). However, a quantitative comparison needs to include the finite directional and temporal resolution in the experiment. We do so by averaging over $N_{\text {eff }}$ statistically independent contributions, which results in $p\left(I, N_{\text {eff }}\right) \propto \theta(Z) Z^{N_{\text {eff }}-1} \exp \left(-Z N_{\text {eff }}\right)$. To determine the coherence $c$, a full statistical analysis of the data is even not necessary. It suffices to evaluate the intensity variance, since, for any exponential distribution, the variance equals the square of the average. Including the $N_{\text {eff }}$ average, we have $c^{2}=N_{\text {eff }} \overline{(I-\bar{I})^{2}} / \bar{I}^{2}$, which is used to determine the coherence. The full curves in Fig. 2 are generated by determining $\bar{I}_{\text {inc }}$ and $\bar{I}_{\text {coh }}$ from average and variance of the data, keeping $N_{\text {eff }}=3$ fixed, which corresponds to an angle and time resolution between one and two speckles.
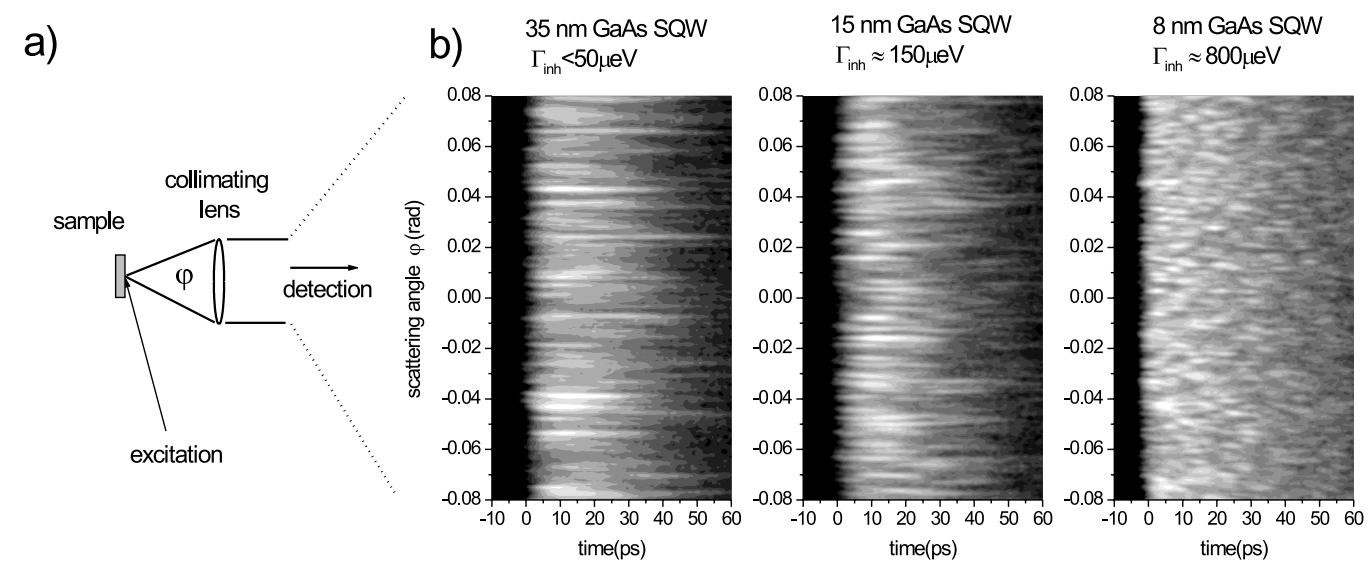

FIG. 1. (a) Schematic experimental geometry. (b) Directionally and temporally resolved emission intensity (2 decades logarithmic grey scale) of a 35 , a 15 , and an $8 \mathrm{~nm} \mathrm{GaAs} \mathrm{SQW}$ at $5 \mathrm{~K}$ and low excitation intensity $\left(\approx 2 \mathrm{~nJ} / \mathrm{cm}^{2}\right.$, exciting an exciton density of $\left.\approx 2 \times 10^{8} \mathrm{~cm}^{-2}\right) . \quad \Gamma_{\text {inh }}$ is the inhomogeneous broadening (FWHM) of the exciton transition measured independently. 

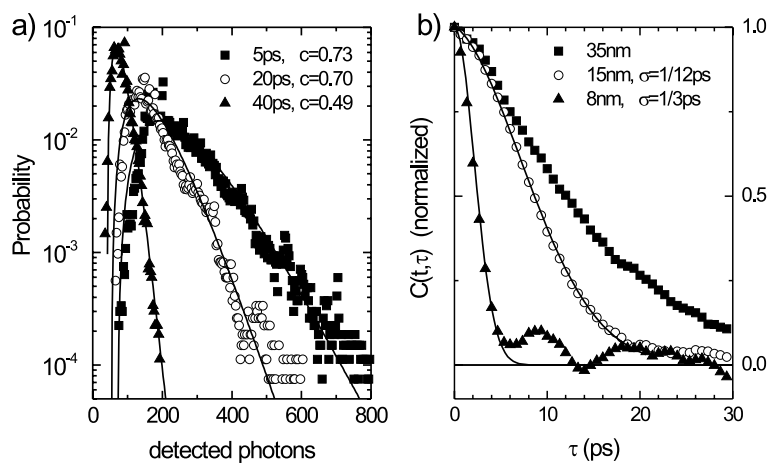

FIG. 2. (a) Intensity statistics of the spatial speckles from a $8 \mathrm{~nm}$ GaAs SQW at various times after excitation, as labeled. Symbols: experimental histogram; full curves: theory with parameters deduced from the data; and $N_{\text {eff }}=3$ throughout. Corresponding coherences $c$ are given in the legend. (b) Intensity correlation function $C(t, \tau)$ at $t=10 \mathrm{ps}$ for different SQWs as a function of time delay $\tau$ (average over about 200 speckles). Lines are according to the discussed model using $\sigma$ as labeled.

As a model for the $1 s$ excitons in a SQW with interface disorder, we consider $N$ localized states within the excitation focus. The state at $R_{j}$ has a transition frequency $\omega_{j}$, the ensemble of which is assumed to be Gaussian distributed (variance $\sigma$ ) and uncorrelated. Each individual state is assigned the same polarization decay rate due to radiative loss and phonon dephasing, $\Gamma=\Gamma_{\text {rad }}+\Gamma_{\text {phon }}$. With these ingredients, a kinetic equation for the (nondiagonal) exciton density matrix [12] is derived and solved for the emitted intensity $I(t)$ after a deltalike excitation pulse at $t=0$.

$$
I(t) \propto e^{-2 \Gamma t} \frac{1}{N}\left|\sum_{j=1}^{N} e^{i q R_{j}-i \omega_{j} t}\right|^{2}+e^{-2 \Gamma_{\mathrm{rad}} t}-e^{-2 \Gamma t} .
$$

The first term represents the coherent part of the emission ( $q$ is the wave vector difference between excitation and detection). Its average over directions is equivalent to an ensemble average and gives the following for large $N$ : $\bar{I}_{\mathrm{coh}}=e^{-2 \Gamma t}\left(1-e^{-\sigma^{2} t^{2}}\right)$. The individual variations over speckles follow an exponential distribution, as explained above. It is the non-self-averaging (or nonergodic [5,13]) feature of Eq. (2), which allows a distinction between the coherent (first term) and incoherent part (last two terms). Within the present model, the coherence is

$$
c=\left(1-e^{-\sigma^{2} t^{2}}\right) /\left(e^{2 \Gamma_{\text {phon }} t}-e^{-\sigma^{2} t^{2}}\right),
$$

which is dominated at times $\sigma t>1$ by an exponential decay with the rate $2 \Gamma_{\text {phon }}$. The radiative decay does not contribute to the loss of coherence.

The temporal speckle dynamics is described by the directionally averaged intensity correlation over the time delay $\tau$,

$$
C(t, \tau)=\frac{\overline{I(t) I(t+\tau)}}{\overline{I(t)} \overline{I(t+\tau)}}-1
$$

which in a time average is known as the temporal secondorder coherence [13]. In the limit $\sigma t>1$, the model gives $C(t, \tau)=\exp \left[-\sigma^{2} \tau^{2}-2 \Gamma_{\text {phon }}(\tau+2 t)\right]$. Measured intensity correlations are displayed in Fig. $2 b$ for the SQWs under study, showing a smaller temporal width for narrower SQWs, in accordance with the larger inhomogeneous broadening.

More generally, all scattering events which remove the excitation out of the ensemble of radiative states, such as radiative recombination or phonon scattering into dark states, contribute to the decay of the emission intensity, characterized by the intensity decay time $T_{1}$ or the related rate $\Gamma_{1}=\left(2 T_{1}\right)^{-1}$ which replaces $\Gamma_{\text {rad }}$ above. In contrast, inelastic scattering in between radiative states leads to a decoherence of the emission, and is quantified by a coherence decay time $T_{2}^{\prime}$ [rate $\Gamma_{2}^{\prime}=\left(T_{2}^{\prime}\right)^{-1}$, replacing $\Gamma_{\text {phon }}$ ]. Backscattering from dark states into radiative states will result in a nonexponential decay of the intensity, and to an increased coherence decay rate. To avoid the influence of these higher-order processes, we analyze the initial decay dynamics only. Both rates together determine the microscopic polarization decay as measured, e.g., in fourwave-mixing experiments, whereas in the present speckle method $\Gamma_{1}$ and $\Gamma_{2}^{\prime}$ can be disentangled easily.

We investigate the temperature dependence of both processes in the $8 \mathrm{~nm} \mathrm{SQW}$. After resonant excitation with pulses of $1.2 \mathrm{ps}$ width, the time evolution of the resonant emission intensity and its coherence is measured and displayed in Fig. 3a. From the coherence analysis, we can deduce the time dynamics of the coherent $\left(\bar{I}_{\text {coh }}\right)$ and incoherent $\left(\bar{I}_{\text {inc }}\right)$ emission intensity, as shown in Fig. $3 b$ for the lattice temperature of $5 \mathrm{~K}$. Here, the intensity decay rate is found to be $2 \Gamma_{1} \approx 1 / 28 \mathrm{ps}$, which is faster than the coherence decay $2 \Gamma_{2}^{\prime} \approx 1 / 75 \mathrm{ps}$. $\Gamma_{1}$ is mainly due to radiative decay, while $\Gamma_{2}^{\prime}$ has to be identified with phonon-assisted dephasing, as shown by the temperature dependence in Fig. 4. For higher temperatures, the intensity and coherence decays get faster (see Fig. 3a). A linear fit of the coherence decay gives $\hbar \Gamma_{2}^{\prime}=(1.9 \pm 0.3) \mu \mathrm{eV}+$ $T(0.46 \pm 0.04) \mu \mathrm{eV} / \mathrm{K}$, while the initial intensity decay is fitted by $\hbar \Gamma_{1}=(8.6 \pm 0.5) \mu \mathrm{eV}+T(0.51 \pm$ $0.04) \mu \mathrm{eV} / \mathrm{K}$. The phonon-mediated scattering within the radiative zone is thus comparable to the outscattering due to phonons. This suggests that the average energy of the interacting acoustic phonons is comparable to the inhomogeneous linewidth. The total acoustic phonon scattering coefficient is $(0.97 \pm 0.1) \mu \mathrm{eV} / \mathrm{K}$ (half width at half maximum), which is smaller than the broadening coefficients of about $2 \mu \mathrm{eV} / \mathrm{K}$ found for the homogeneous width in wide GaAs quantum wells [14]. The intensity decay at the lowest temperatures is presumably dominated by the radiative decay with $T_{1}=38 \pm 5$ ps.

In general, we observe slower dynamics and longer coherence times than reported [2,3,5], which we relate to the multiple QW structures and the larger exciton densities used. The results of [1] are in better agreement with 


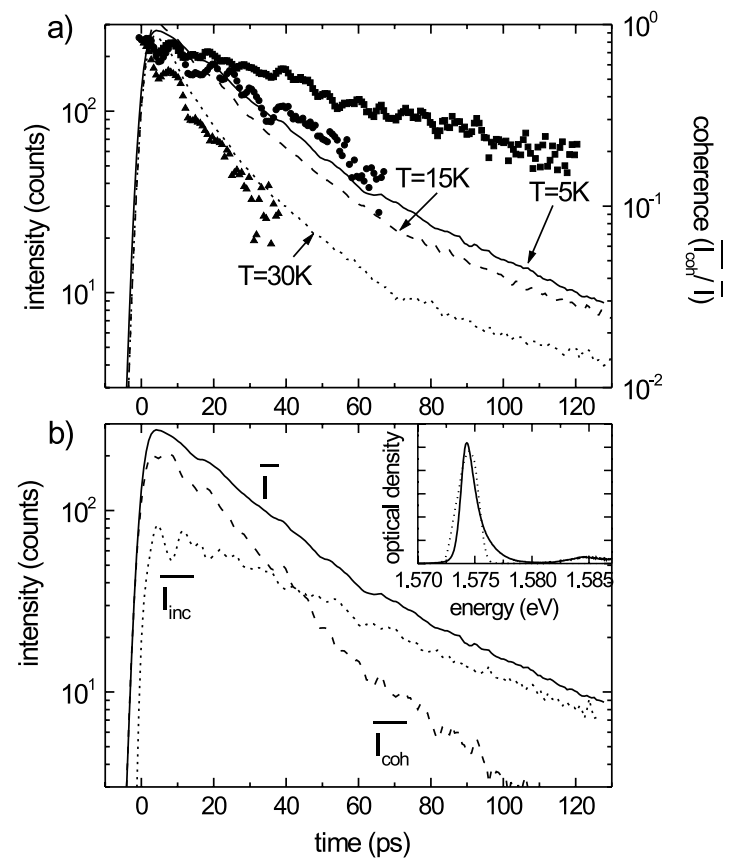

FIG. 3. Secondary emission intensity and its coherence from the $8 \mathrm{~nm}$ GaAs SQW for different lattice temperatures. (a) Angular averaged total intensity $\bar{I}$ for $T=5,15$, and $30 \mathrm{~K}$ (lines), and the corresponding coherence $c$ (symbols) as a function of time. (b) The measured total intensity $\bar{I}$ (full curve) at $T=5 \mathrm{~K}$ is split into its coherent (dashed curve) and incoherent parts (dotted curve) using the coherence from Fig. 5a. Inset: Optical density of the excitonic region, deduced from the measured luminescence at $30 \mathrm{~K}$ assuming a Boltzmann distribution of excitons. Dotted curve: spectral pulse shape.

our findings. However, we found that the polarization assumptions used are not valid for larger inhomogeneous broadenings.

In conclusion, we have presented a novel approach of measuring the coherence of secondary emission which relies on the analysis of the spatial speckle pattern. Being a linear optical method, it allows one to determine the coherence in a quantitative manner down to low intensities, and without the need of an interferometric setup. For the investigated secondary emission of inhomogeneously broadened excitons in quantum wells, the decoherence rate (phonon scattering) and the intensity decay are measured independently. This represents a clear advantage over two-beam four-wave-mixing experiments where only the sum of both (total "dephasing rate") is determined. The coherence of the emission is not influenced by the lifetime, and the pure dephasing time $T_{2}^{\prime}$ is found to exceed significantly the lifetime $T_{1}$ at low temperatures in the investigated samples.

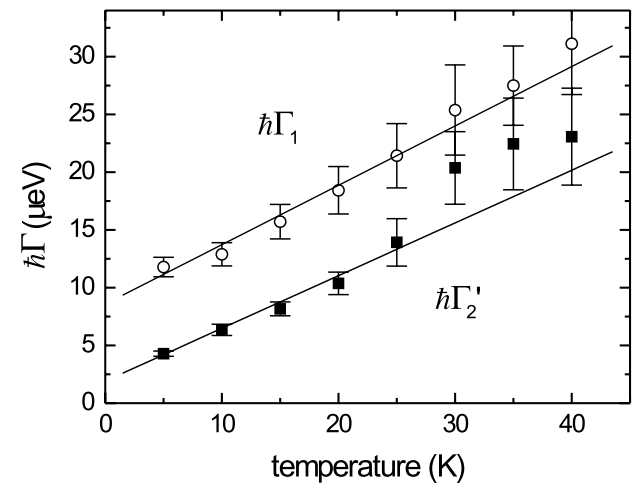

FIG. 4. Lifetime rate $\hbar \Gamma_{1}$ (circles) and decoherence rate $\hbar \Gamma_{2}^{\prime}$ (squares) for the $8 \mathrm{~nm} \mathrm{GaAs} \mathrm{SQW} \mathrm{as} \mathrm{a} \mathrm{function} \mathrm{of} \mathrm{temperature.}$

The authors thank J. Riis Jensen and C. B. Sørensen (III-V Nanolab) for growing the high-quality GaAs samples. This work was supported by the Danish Ministries of Research and Industry in the framework of CNAST, and by the German Science Foundation (DFG) within the "Schwerpunktprogramm Quantenkohärenz in Halbleitern."

[1] H. Stolz, D. Schwarze, W. von der Osten, and G. Weimann, Phys. Rev. B 47, 9669 (1993).

[2] H. Wang, J. Shah, T. Damen, and L. Pfeiffer, Phys. Rev. Lett. 74, 3065 (1995).

[3] S. Haacke, R. A. Taylor, R. Zimmermann, I. Bar-Joseph, and B. Deveaud, Phys. Rev. Lett. 78, 2228 (1997).

[4] M. Gurioli, F. Bogani, S. Ceccherini, and M. Colocci, Phys. Rev. Lett. 78, 3205 (1997).

[5] D. Birkedal and J. Shah, Phys. Rev. Lett. 81, 2372 (1998).

[6] M. Wörner and J. Shah, Phys. Rev. Lett. 81, 4208 (1998).

[7] L. Lepetit, G. Chériaux, and M. Joffre, J. Opt. Soc. Am. B 12, 2467 (1995).

[8] B. Chu, Laser Light Scattering (Academic Press, New York, 1974); J.C. Dainty, Laser Speckle and Related Phenomena (Springer-Verlag, New York, 1984).

[9] M. Tomita and T. Matsumoto, J. Opt. Soc. Am. B 12, 170 (1995).

[10] W. Demtröder, Laser Spectroscopy (Springer-Verlag, Berlin, 1977).

[11] F. T. Arecchi, M. Giglio, and U. Tartari, Phys. Rev. 163, 187 (1967).

[12] R. Zimmermann, Nuovo Cimento Soc. Ital. Fis. 17D, 1801 (1995).

[13] R. Loudon, The Quantum Theory of Light (Oxford Science Publications, Oxford, 1983).

[14] D. Gammon et al., Phys. Rev. B 51, 16785 (1995). 